\title{
A retrospective clinicopathological analysis of small-cell carcinoma of the uterine cervix
}

\author{
SAMINA DONGOL ${ }^{1}$, YANHONG TAI ${ }^{2}$, YI SHAO $^{3}$, JIE JIANG ${ }^{1}$ and BEIHUA KONG ${ }^{1}$ \\ ${ }^{1}$ Department of Obstetrics and Gynecology, Qilu Hospital of Shandong University; ${ }^{2}$ Jinan Military Area General Hospital; \\ ${ }^{3}$ Shandong University School of Medicine, Jinan, Shandong, P.R. China
}

Received June 6, 2013; Accepted August 28, 2013

DOI: $10.3892 / \mathrm{mco} .2013 .193$

\begin{abstract}
Cervical cancer encompasses several histological types, including neuroendocrine tumors (NETs). Small-cell carcinoma of the uterine cervix (SCCC) is the most common and aggressive subtype of cervical NET. The objective of this case report was to investigate SCCC using a retrospective clinicopathological approach. Four cases of large $(\geq 4 \mathrm{~cm})$ SCCCs are presented in this case study. The patients were diagnosed with SCCC through a sequential hierarchy of physical examinations, laboratory reports, radiological reports, immunohistochemical and pathological tests. The diagnosis for each case was made at various stages (Ib1, Ib2, IIa2 and IIb, according to the FIGO staging system, 2000) and each of the patients received different multimodality therapeutic regimens. All the patients underwent radical hysterectomy and pelvic lymphadenectomy, followed by adjuvant chemotherapy. Neoadjuvant chemotherapy was administered prior to surgery in two of the patients. The clinical and pathological analyses were assessed using a retrospective measure, maintaining timely follow-ups. SCCC is a rare but serious gynecological malignancy. This condition has a poor prognosis due to its high aggressiveness, high rate of metastases and mortality. Furthermore, the rarity of this disease represents a hindrance to adequate research and development of novel, efficient therapeutic regimens.
\end{abstract}

\section{Introduction}

Cervical cancer is a major entity in the field of gynecological oncology, encompassing several histological types, among which neuroendocrine tumors (NETs) represent a small fraction. NETs are commonly found in the gastrointestinal system and the lungs; however, they are rarely encountered

Correspondence to: Dr Jie Jiang, Department of Obstetrics and Gynecology, Qilu Hospital, Shandong University, 107 Wenhua West Road, Jinan, Shandong 250012, P.R. China

E-mail: qljiangjie@sdu.edu.cn

Key words: small-cell carcinoma of cervix, multimodal treatment regimen, neoadjuvant chemotherapy, prognostic factor in the uterine cervix. NETs are classified into four distinct histological types: small-cell carcinoma, large-cell carcinoma, classical carcinoid tumor and atypical carcinoid tumor (1). Small-cell carcinoma of the cervix (SCCC) is the most common and aggressive subtype of cervical NET. Its high degree of aggressiveness and early distant metastatic spread accounts for the poor prognosis of SCCC at the time of diagnosis. The 5-year survival rate of early-stage SCCC may range from 30 to $46 \%$, whereas for advanced-stage tumors it may be as low as $0-15 \%$ (2). The therapeutic approach for this type of malignancy represents a clinical challenge. The therapeutic models are largely extrapolated from those for small-cell lung cancer (SCLC) (3). In addition, patients treated with modalities similar to those for other common types of cervical cancer exhibited a poorer prognosis (4).

SCCC is similar to the small-cell cancer arising from the pulmonary bronchi and was first described by Reagan et al (5). However, although this malignancy resembles SCLC, the two exhibit varied degrees of immunohistochemical reactivity and respond diversely to the same multimodality regimens. Since its identification, SCCC has been extensively investigated in order to ascertain a better understanding of this disease and design more effective therapeutic regimens. Currently, the focus is shifted towards elucidating the causative factors and the prognostic aspect of this condition. Due to the rarity of SCCC, it is difficult to gather sufficient information in order to assess the evidence-based data regarding its therapeutic and prognostic aspects. This is also hindering any progress towards conducting clinical trials or investigations on a larger scale. Thus, retrospective case studies may prove to be a valuable tool in furnishing information on SCCC, which may contribute to a better understanding of this malignancy.

\section{Patients and methods}

Cases. In this study we present four cases of SCCC in Chinese patients aged 14-43 years. The tumors were staged as Ib1, Ib2, IIa2 and IIb, according to the FIGO staging system, 2000. Each patient was admitted to the hospital with the common symptom of irregular vaginal bleeding, with or without lower abdominal pain. One of the patients was in her early teens, with no sexual history. The patient was initially diagnosed with adenocarcinoma on preliminary biopsy, which was later identified as compound adenocarcinoma with neuroendocrine 
components. Another patient was on the 32 nd week of her first gestation. Immunohistochemical tests were performed for the following tumor markers: neuron-specific enolase (NSE), chromogranin A (CgA), cytokeratin (CK), CD99, synaptophysin (Syn), epithelial membrane antigen and CD117. Chest X-rays, CT and MRI scans were ordered to determine the extent of tumor spread and detect any distant metastases.

Neoadjuvant treatment. Following admittance to the hospital, the patients were approached with different therapeutic regimens. Except for the patient who was in the third trimester of gestation, the remaining three patients received neoadjuvant chemotherapy. One patient received 3 cycles of etoposide and platinum (PE) and the second patient was administered 2 cycles of cisplatin, vincristine and bleomycin (PVB). The third patient, who was initially diagnosed with adenocarcinoma on biopsy, underwent 4 cycles of transcatheter arterial chemoembolization with carboplatin, vincristine and mitomycin.

Surgical treatment. One patient underwent laparoscopic radical hysterectomy and radical hysterectomy with pelvic lymphadenectomy was performed on two patients, one of whom had an additional unilateral adnexal resection. Cesarean section was performed on the patient who was in the 33rd week of gestation, followed by radical hysterectomy with bilateral salpingo-oophorectomy and pelvic and periaortic lymphadenectomy.

Adjuvant treatment. Following hysterectomy, adjuvant chemotherapy with the PE regimen was administered to two patients, including the patient who had received PE as adjuvant chemotherapy and the patient who was initially diagnosed with adenocarcinoma, for 3 and 5 cycles, respectively. Four cycles of the PVB regimen were administered to the third patient, whereas the patient who underwent the Cesarean section received 3 cycles of adjuvant chemotherapy with platinum and paclitaxel (Taxol).

\section{Results}

Gynecological examination. The gynecological examinations revealed cervical growths $>4 \mathrm{~cm}$ in diameter (Fig. 1). Pelvic wall infiltration was evident in two of the cases and no bilateral inguinal or femoral lymph node enlargement was detected. The presence of the tumors was also confirmed through ultrasonograms. The tumors were biopsied during a colposcopical examination and the pathological diagnosis was confirmed by pathologists specializing in the field of gynecological oncology as SCCC in three of the cases (Fig. 2), two of which were purely neuroendocrine, whereas one exhibited components of small-cell carcinoma combined with adenocarcinoma. The fourth case, however, was initially diagnosed as cervical adenocarcinoma after the biopsy and was only diagnosed as SCCC after the results of the pathological examination of the surgically resected specimen.

Radiological assessment and immunohistochemical staining. The radiological assessment of one of the cases demonstrated cervical lesions with possible lymph node metastasis. However, none of the cases presented with metastatic lesions on cranial CT, chest CT or ultrasonograms of the hepatobiliary system.

The immunohistochemical staining revealed a predominance of NSE, Syn and CgA. Only one case exhibited partially positive reactivity for CD117 (Fig. 3A-C). The youngest subject in this study presented with a significantly advanced tumor stage (IIb), with paracervical infiltration and unilateral obturator and internal iliac lymph node metastasis, whereas no lymph node involvement was identified in the any of the other cases.

Follow-up. During the regular follow-ups, all the patients developed varied degrees of complications. Despite several attempts, follow-up was not possible for one of the cases. Another patient developed acute renal failure, for which she received timely dialysis. The patient declined any further interventions and was discharged on her own terms. On follow-up, we were informed that the patient had succumbed to renal complications 7 months after her initial diagnosis. The patient who was pregnant at the time of diagnosis developed a liver metastasis on radiological examination shortly after the operation and succumbed to acute renal complications 14 months after her initial diagnosis. The youngest patient achieved a remarkable recovery despite her advanced-stage disease and lymph node metastasis. The patient only exhibited a mild allergic reaction to the adjuvant chemotherapy, which was managed symptomatically, with full recovery.

\section{Discussion}

The aggressive malignant behavior with the propensity to metastasize is the hallmark of NETs and it is generally associated with a poor prognosis. The tumor advances early and at the time of diagnosis it is usually widely disseminated, with bone, brain, liver and bone marrow being the most common sites of metastasis. Previous studies reported a high rate of lymph node metastasis in patients with disease that is apparently confined to the cervix (4-6). Among the different types of cervical NETs, SCCC is the most common and the most severe. The rate of prevalence, however, is as low as $0.5-5 \%$ of all cervical malignancies (5-7). The rarity of this condition confers a high level of complexity in ascertaining a target population to conduct any clinical trials. Thus far, treatment regimens for NETs of the cervix have been based on small case series of patients, leaving a lot to be unveiled regarding various aspects of this disease. There have been several hypotheses regarding the origin of this type of tumor, one of which suggested that its origin resides with a minority of argyrophilic cells already present in the cervical epithelium, whereas others suggested that SCCCs originate from the pleuripotent neuroendocrine cells in the cervical epithelium and some even believe that they may originate from the transformation of the uterine columnar epithelial cells into neuroendocrine cells (7,24-26).

The poor prognosis at the time of diagnosis is attributed to the extremely aggressive nature and early progression of SCCCs. Several factors have been implicated in prognosis, among which tumor stage has exhibited a statistically significant correlation with disease prognosis, according to previous studies $(2,6,8)$. In addition, patients with SCCC should also undergo bone, liver and brain scanning, as well as bone 


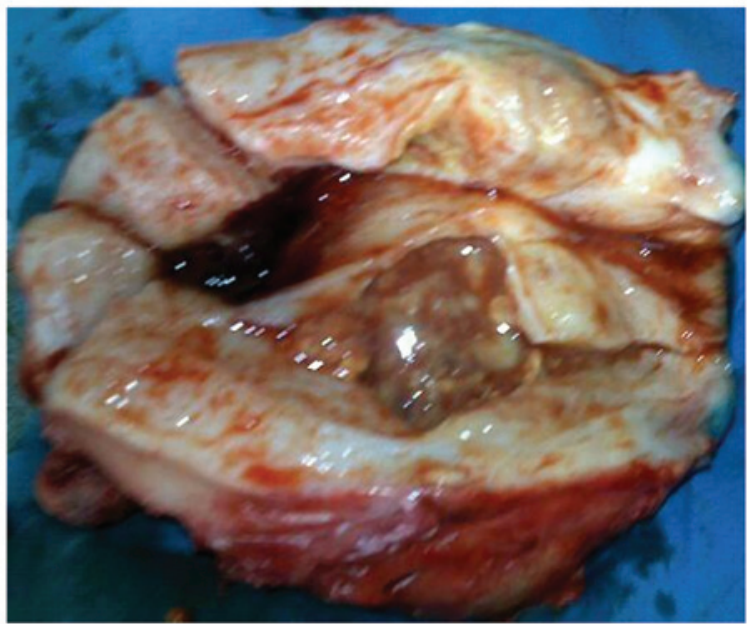

Figure 1. Resected uterus exhibiting a tumor growth $(\geq 5 \mathrm{~cm})$.

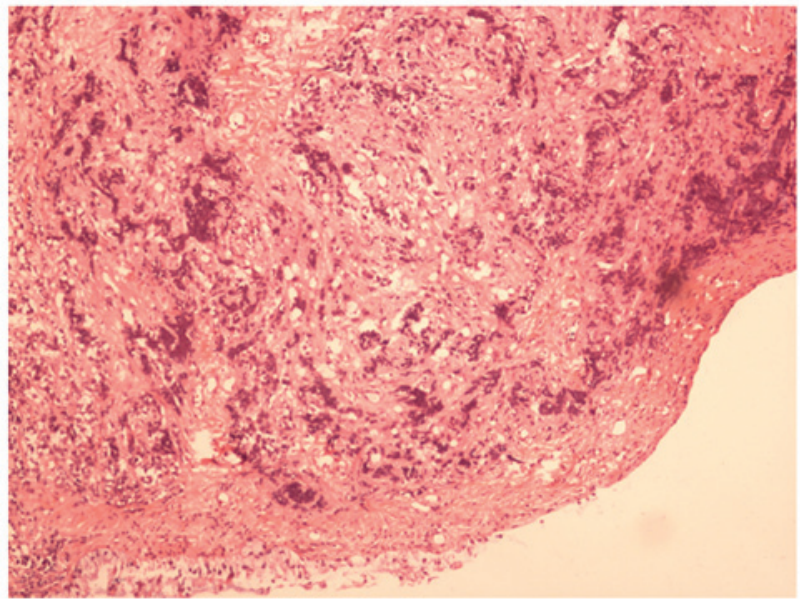

Figure 2. Histological section of biopsy specimen. Hematoxylin and eosin staining (magnification, $\mathrm{x} 400$ ). The biopsy demonstrated a dense population of cells with hyperchromatic nuclei and scant cytoplasm, which is similar to the histological characteristics of small-cell carcinoma of the lungs.

marrow aspiration and biopsy, in order to evaluate the possibility of metastasis, since this is also responsible for the poor prognosis of SCCC. Although other factors, such as tumor size, age, smoking and histological type, were shown to exert an obvious effect on prognosis, they were found to be statistically insignificant $(2,8)$. According to several studies, the 5-year survival rate was $\leq 50 \%$ in patients who were administered multidisciplinary treatment (2-6). In cases where the patients were diagnosed with advanced-stage disease, the survival time averaged 2 years.

The diagnosis of SCCC is based on a set of cytological, pathological, immunohistochemical and radiological tests. Pathologically, the diagnosis is supported by the identification of neuroendocrine granules on electron microscopy, as well as by immunoperoxidase studies that are positive for a variety of neuroendocrine proteins, such as calcitonin, insulin, glucagon, somatostatin, gastrin and adrenocorticotropic hormone (ACTH). On pathological examination, the tumor may present as a pure histological type or with an admixture of characteristics of adenocarcinoma or squamous cell carci-
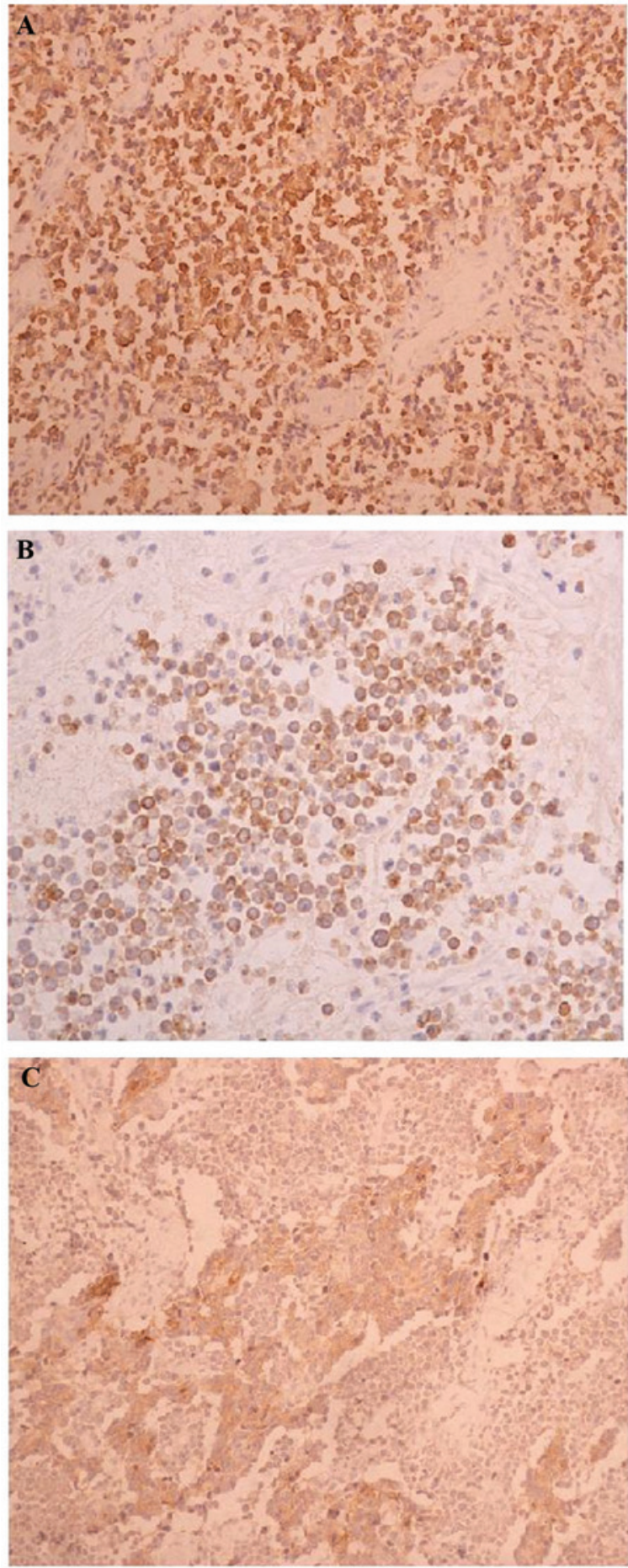

Figure 3. Immunohistochemical finding of biopsy specimens (magnification, $\mathrm{x} 400$ ). (A) Immunostaining for neuron-specific enolase revealed a positive reaction. (B) Immunostaining showing a positive reaction for synaptophysin. (C) Immunostaining for CD117 showing partial positive reactivity.

noma. This may occasionally disguise the true nature of the tumor, making pathological detection more challenging (9). SCCCs were shown to exhibit definite immunohistochemical characteristics, which form the basis for its diagnosis. Out of several neuroendocrine and epithelial markers, NSE, CgA, Syn, CK and CD56 are the most prominent, frequently detected in this type of tumors $(10,14,22-24)$. Over the last few years, studies were conducted on the CD117 marker (c-kit proto-oncogene, a tyrosine kinase) which is overexpressed 

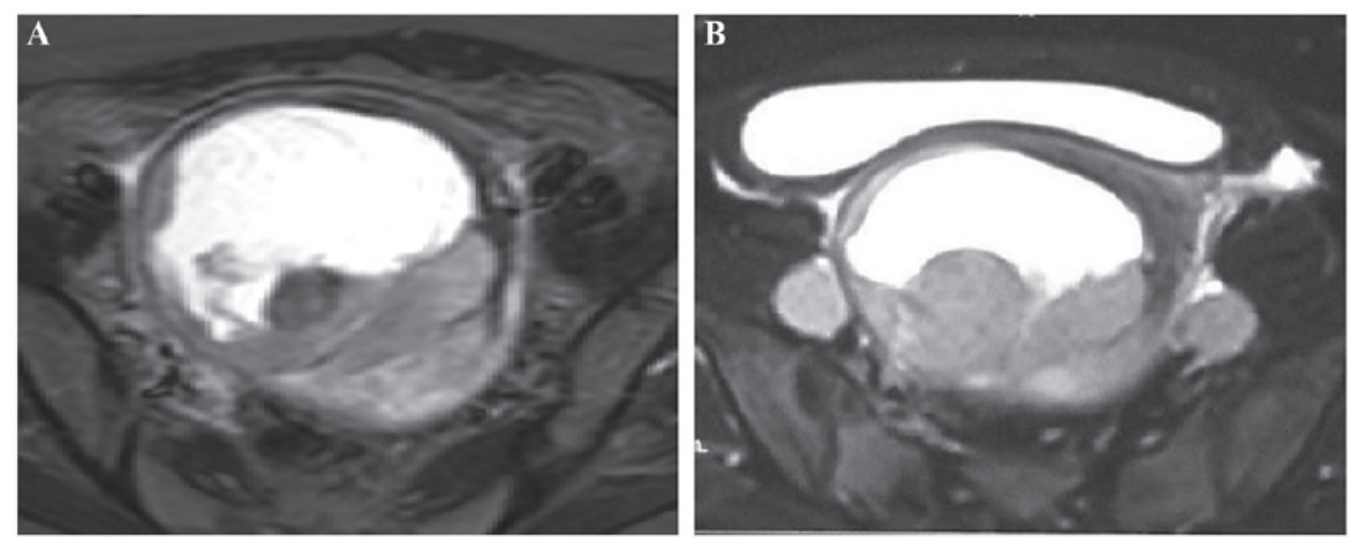

Figure 4. T2-weighted magnetic resonance images of the tumor: (A) tumor after the initial diagnosis and (B) tumor after the patient received 2 cycles of neoadjuvant chemotherapy.

in the majority of SCLC cases and has set a foundation for targeted therapy $(11,12)$. Although previous findings regarding the expression of CD117 in SCCC failed to provide conclusive results, there are ample possibilities for other markers specific to SCCC that may eventually lay the foundation for designing an efficient targeted therapy.

Since even patients with early-stage disease may develop distant metastases, multimodality treatment is clearly a more effective approach to the management of $\operatorname{SCCC}(8,13,14)$. The principle treatment regimen is mainly deduced from that for SCLC, owing to the histological similarity between the two entities. Pazdur et al (15) first suggested the application of a chemotherapeutic regimen for SCCC similar to that used for SCLC. However, an optimal treatment regimen specific to SCCC has not been established. Radical surgery with lymphadenectomy and lymph node sampling may be optimal for small tumors at early stages, sized $<2 \mathrm{~cm}$, whereas neoadjuvant chemotherapy is recommended for debulking large tumors ( $>4 \mathrm{~cm}$ ) (Fig. 4A and B) in order to improve their resectability $(13,14,16)$. Adjuvant chemoradiotherapy administered postoperatively was shown to produce better outcomes $(10,12)$. The main active chemotherapeutic agent of choice for small-cell carcinoma is etoposide. Zivanovic et al (17) hypothesized that etoposide-based chemotherapy may prevent distant metastasis in patients with early-stage disease. In a single-case study by Futagami et al (18), long-term survival was achieved by a chemotherapeutic combination of platinum with etoposide in a patient with early-stage disease. Bermudez et al (14) suggested neoadjuvant chemotherapy with vincristine, bleomycin and platinum in their study. More recent studies introduced novel neoadjuvant chemotherapeutic regimens, which demonstrated promising results. Nasu et al (3) reported the efficacy of a combination of irinotecan and cisplatin as neoadjuvant chemotherapy in a case with advanced SCCC, which is also the therapeutic protocol for advanced SCLC. Similarly, other studies suggested paclitaxel and platinum as the fundamental therapeutic regimen $(13,18)$. Platinum-based chemotherapy was shown to be significantly beneficial and is thus considered as the first-line chemotherapeutic regimen $(16,19)$. Other combination regimens may include vincristine, adriamycin and cyclophosphamide. A study conducted by Embry et al (19) reported an improvement in the survival rates of patients with large-cell NETs undergoing perioperative chemotherapy including platinum, with or without etoposide. Recurrent tumors are commonly treated with a combination of paclitaxel and cisplatin.

The patients in our study developed various complications at various time points during their chemotherapy administration. Therefore, therapeutic strategies aimed at minimizing such complications may improve survival rates. Prophylactic cranial irradiation has been included in the postoperative treatment protocol in SCLC patients and has been optionally incorporated into the treatment of SCCC. Para-aortic lymph node involvement is a rather frequent finding; thus, para-aortic irradiation has also been introduced into the therapeutic measure (18).

There has been a paradigm shift in the preventive aspect of this disease over the last few years. Siriaunkgul et al (20) reported that $75 \%$ of the cases of cervical NETs were positive for HPV 18 and 30\% were positive for HPV 16, both of which are high-risk HPV variants. Thus, it was hypothesized that vaccination against HPV 16 and 18 may represent a preventive measure $(20,21)$. However, no further related studies are currently available.

In conclusion, SCCC is a highly detrimental and aggressive type of cervical malignancy. Available data regarding various aspects of this type of tumor are limited and extensive studies are required to achieve a better understanding of this disease. Further investigations on the subject matters we proposed in our present study may also provide a route towards novel findings regarding the efficient management of this tumor.

\section{Acknowledgements}

This study was partially supported by the National Natural Science Foundation of China (81072121, 81372808 and 81173614 ) and also by the Science and Technology Development Planning Foundation (2012G0021823 and 2011GSF12122).

\section{References}

1. Albores-Saavedra J, Gersell D, Gilks CB, et al: Terminology of endocrine tumors of the uterine cervix: results of a workshop sponsored by the college of American Pathologists and the National Cancer Institute. Arch Pathol Lab Med 121: 34-39, 1997. 
2. Chan JK,Loizzi V,Burger RA, Rutgers J and Monk BJ: Prognostic factors in neuroendocrine small cell cervical carcinoma, a multivariate analysis. Cancer 97: 568-574, 2003.

3. Nasu K, Hirakawa T, Okamoto M, Nishida M, Kiyoshima C Matsumoto H, Takai N and Narahara H: Advanced small cel carcinoma of the uterine cervix treated by neoadjuvant chemotherapy with irinotecan and cisplatin followed by radical surgery. Rare Tumors 3: e6, 2011.

4. Sheets EE, Berman ML, Hrountas CK, et al: Surgically treated, early-stage neuroendocrine small-cell cervical carcinoma. Obstet Gynecol 71: 10-14, 1988.

5. Reagan JW, Hamonic MJ and Wentz WB: Analytical study of the cells in cervical squamous-cell cancer. Lab Invest 6: 241-250, 1957.

6. Boruta DM 2nd, Schorge JO, Duska LA, Crum CP, Castrillon DH and Sheets EE: Multimodality therapy in early-stage neuroendocrine carcinoma of the uterine cervix. Gynecol Oncol 81: 82-87, 2001.

7. Barrett RJ 2nd, Davos I, Leuchter RS and Lagasse LD: Neuroendocrine features in poorly differentiated and undifferentiated carcinomas of the cervix. Cancer 60: 2325-2330, 1987.

8. Chang TC, Lai CH, Tseng CJ, Hsueh S, Huang KG and Chou HH Prognostic factors in surgically treated small cell cervical carcinoma followed by adjuvant chemotherapy. Cancer 83 712-718, 1998

9. Gilks CB, Young RH, Gersell DJ and Clement PB: Large cell neuroendocrine carcinoma of the uterine cervix: a clinicopathologic study of 12 cases. Am J Surg Pathol 21: 905-914, 1997.

10. Giorgaze T, Kanhere R, Pang C, Ganote C, Miller LE, Tabaczka P, Brown E and Husain M: Small cell carcinoma of the cervix in liquid-based Pap test: utilization of split-sample immunocytochemical and molecular analysis. Diagn Cytopathol 40: 214-219, 2012.

11. Wang HL and Lu DW: Overexpression of c-kit protein is an infrequent event in small cell carcinomas of the uterine cervix. Mod Pathol 17: 732-738, 2004.

12. Sukpan K, Settakorn J, Khunamornpong S, Cheewakriangkrai C, Srisomboon J and Siriaunkgul S: Expression of survivin, CD117, and C-erbB-2 in neuroendocrine carcinoma of the uterine cervix. Int J Gynecol Cancer 21: 911-917, 2011.

13. Dueñas-Gonzalez A, López-Graniel C, González-Enciso A, Cetina L, Rivera L, Mariscal I, Montalvo G, Gómez E, de la Garza J, Chanona G and Mohar A: A phase II study of multimodality treatment for locally advanced cervical cancer: neoadjuvant carboplatin and paclitaxel followed by radical hysterectomy and adjuvant cisplatin chemoradiation. Ann Oncol 14: 1278-1284, 2003.

14. Bermudez A, Vighi S, Garcia A and Sardi J: Neuroendocrine cervical carcinoma: a diagnostic and therapeutic challenge. Gynecol Oncol 82: 32-39, 2001.
15. Pazdur R, Bonomi P, Slayton R, et al: Neuroendocrine carcinoma of the cervix: implications for staging and therapy. Gynecol Oncol 12: 120-128, 1981.

16. Hoskins PJ, Swenerton KD, Pike JA, et al: Small-cell carcinoma of the cervix: fourteen years of experience at a single institution using a combined-modality regimen of involved-field irradiation and platinum-based combination chemotherapy. J Clin Oncol 21: 3495-3501, 2003

17. Zivanovic O, Leitao MM Jr, Park KJ, et al: Small cell neuroendocrine carcinoma of the cervix: Analysis of outcome, recurrence pattern and the impact of platinum-based combination chemotherapy. Gynecol Oncol 112: 590-593, 2009.

18. Futagami M, Yokoyama Y and Mizunuma H: Presentation of a patient with pT2bN1M0 small cell carcinoma of the uterine cervix who obtained long-term survival with maintenance chemotherapy, and literature-based discussion. Eur J Gynaecol Oncol 32: 99-102, 2011

19. Embry JR, Kelly MG, Post MD and Spillman MA: Large cell neuroendocrine carcinoma of the cervix: prognostic factors and survival advantage with platinum chemotherapy. Gynecol Oncol 120: 444-448, 2011.

20. Siriaunkgul S, Utaipat U, Settakorn J, Sukpan K, Srisomboon J and Khunamornpong S: HPV genotyping in neuroendocrine carcinoma of the uterine cervix in northern Thailand. Int J Gynaecol Obstet 115: 175-179, 2011.

21. Bian LH, Wang XL, Guo YF, Wu XZ, Song L and Liu HT: Study of human papillomavirus in small cell neuroendocrine carcinoma of the uterine cervix. Chin J Exp Clin Virol 25: 63-65, 2011 (In Chinese).

22. Rashed MM and Bekele A: Neuroendocrine differentiation in a case of cervical cancer. Pan Afr Med J 6: 4, 2010.

23. Conner MG, Richter H, Moran CA, Hameed A and Albores-Saavedra J: Small cell carcinoma of the cervix: a clinicopathologic and immunohistochemical study of 23 cases. Ann Diagn Pathol 6: 345-348, 2002

24. Deng GH, Zhang X and Wu LY: Clinicopathological analysis of nine cases of small cell carcinoma of the uterine cervix. Chin J Oncol 32: 199-202, 2010 (In Chinese).

25. Cohen JG, Kapp DS, Shin JY, Urban R, Sherman AE, Chen LM, Osann K and Chan JK: Small cell carcinoma of the cervix: treatment and survival outcomes of 188 patients. Am J Obstet Gynecol 203: 347, 2010 .

26. Horn LC, Fischer U and Bilek K: Neuroendocrine differentiated carcinoma of the uterine cervix. Zentralbl Gynakol 118: 679-683, 1996 (In German). 